\title{
HARMONIC INVERSIONS IN PG $(4,3)$ AND SOME RELATED CONFIGURATIONS
}

\author{
NIRMAL KUMAR BASU \\ (Received 15 December 1969; revised 2 November 1970) \\ Communicated by G. Szekeres
}

\section{Introduction}

Geometries of one, two and three dimensional spaces over GF(3) have been considered in [2]. A line in a space over $G F(3)$ contains 4 points and every pair of points is harmonic to its complementary pair. This feature makes the transformation of harmonic inversion interesting in a space over GF(3).

In a space of four dimensions, a 3-dimensional subspace will be called a solid. Given a point $P$ and a solid $s$ not passing through $P$, the harmonic conjugate of any point $X$ with respect to $P$ and the point where $P X$ meets $s$ is called the harmonic inverse of $X$ with respect to $P$ and $s$. In 4-space, the transformation of harmonic inversion may also be defined with respect to a line and a plane as fundamental. That is, given a line $l$ and a plane $p$ not meeting $l$, there is a line $m$ through any point $X$ meeting $l$ and $p$. The harmonic conjugate of $X$ with respect to the points where $m$ meets $l$ and $p$ will also be called the harmonic inverse of $X$ with respect to $l$ and $p$. The transformation of harmonic inversion is a projective transformation. For distinction, we shall denote these two different transformations as harmonic inversion $(P, s)$ and harmonic inversion $(l, p)$ respectively. The object of this paper is to consider the harmonic inverses of a point with respect to the different pairs of opposite elements of a 4-simplex in $P G(4,3)$ and then obtain some familiar as well as new configurations. The elements of $G F(3)$ are taken as $0,1,-1$, where $1+1=-1$.

\section{Harmonic Inversion $(1, p)$}

We take a 4-dimensional simplex, and as an edge and the opposite plane of such a simplex do not meet, we can consider the harmonic inverses of a point with respect to such a pair of elements. We take a given simplex as the simplex of reference $A_{1} A_{2} A_{3} A_{4} A_{5}$ and a given point as the unit point $X(1,1,1,1,1$, and we shall now determine the ten harmonic inverses of $X$ with respect to all the edges and opposite planes. 
The points on an edge $A_{i} A_{j}$ other than $A_{i} A_{j}$ are and only two, i.e. $A_{i} \pm A_{j}$, whose co-ordinates in the $i$-th positions $(i<j)$ may be taken as 1 and in the $j$-th position is either 1 or -1 ; the other co-ordinates are all zero. The point $A_{i}+A_{j}$ on $A_{i} A_{j}$ whose $i$-th and $j$-th co-ordinates are equal is denoted as $Q_{i j}$. The plane $p_{i j}$ of the simplex opposite to $A_{i} A_{j}$ is $x_{i}=0=x_{j}$. Thus it follows that the line $X Q_{i j}$ is the line through $X$ which meets $A_{i} A_{j}$ and $p_{i j}$. The harmonic conjugate of $X$ with respect to $Q_{i j}$ and $p_{i j} \cdot X Q_{i j}$ is the remaining point on $X Q_{i j}$, i.e. $X+Q_{i j}$, whose $i$-th and $j$-th co-ordinates are each -1 and the other co-ordinates are all 1 . Of course, a point in a space ovef $G F(3)$ may be represented by two sets of coordinates which are negatives of each other.

The ten harmonic inverses $P_{i j}(i<j ; i, j=1,2,3,4,5)$ of $X$ with respect to the edges $A_{i} A_{j}$ and opposite planes $p_{i j}$ of the simplex $A_{1} A_{2} A_{3} A_{4} A_{5}$ and the 5 vertices $A_{i}, i=1,2, \ldots 5$ from a configuration of fifteen points and fifteen lines in $P G(4,3)$ where each line contains three points. For, the sets of collinear points are

where

$$
P_{i j}, P_{l m}, A_{k}
$$

$$
i, j \neq l, \quad m \neq k, \quad i<j, \quad l<m .
$$

Thus these fifteen points form Segre's well-known $15_{3}$ configuration [1]. Hence in $P G(4,3)$, Segre's configuration can be obtained in a manner quite different from the usual methods [1].

\section{Harmonic Inversion $(P, s)$}

2.1. We shall now consider the harmonic inverses of a point with respect to the vertices and opposite solids of a given simplex. We take the given simplex as the simplex of reference $A_{1} A_{2} A_{3} A_{4} A_{5}$ and the point as the unit point $X(1,1,1,1,1)$. The harmonic conjugate of $X$ with respect to the vertex $A_{i}$ and $A_{i} X \cdot S_{i}$ where $S_{i}$ is the solid face of the simplex opposite to $A_{i}$, is the point $P_{i}$ whose $i$-th coordinate is -1 and each of the other coordinates is 1 .

Thus the five harmonic inverses $P_{i}(i=1,2,3,4,5)$ of $X$ are obtained and it is found in $P G(4,3)$ that the points $P_{i}$ lie on the solid

$$
x_{0}+x_{1}+x_{2}+x_{3}+x_{4}=0
$$

which is the polar solid of $X$ for the simplex of reference [4].

2. 2. We now consider any set of six points in $P G(4,3)$ no five of which lie on a solid. Let the six points be denoted as $A_{i}, i=1,2,3,4,5,6$. Now, the symbols of these six points must be connected by a syzygy which by proper choice of the symbols may be supposed to be (see [1])

$$
A_{1}+A_{2}+A_{3}+A_{4}+A_{5}+A_{6}=0 \text {. }
$$

After this no further multiplication by -1 of these symbols, if not all, is proper. 
Any five of these six points determine a simplex and we now want to determine the polar solid of any of these six points with respect to the simplex determined by the remaining five. The polar solid of any point $A_{i}$ with respect to the simplex determined by the other five points contains the ten points $A_{j}-A_{k}(j, k \neq i)[3]$ and is determined by 4 independent points of this type by fixing either $j$ or $k$. If we take the simplex determined by some five points $A_{i}$ as the simplex of reference and the remaining point as the unit point, then it can easily be verified that all the fifteen points $A_{j}-A_{k}(j, k=1,2,3,4,5,6)$ lie on the same solid

$$
x_{0}+x_{1}+x_{2}+x_{3}+x_{6}=0 \text {. }
$$

Thus it follows that given a set of six points in $P G(4,3)$ no five of which lie on a solid, the polar solid of any point for the simplex determined by the remaining five points is the same for all the points of the set.

23. Court's conjecture on $n+2$ points in [ $n]$ as proved by Mandan in [3] is as follows:

Let $A_{i}(i=0,1,2 \ldots n+1)$ be $n+2$ distinct points in $[n]$ such that no $n+1$ of them lie in a prime and therefore any $n+1$ of them form the $n+1$ vertices $A_{j}$ of a simplex $S\left(A_{j}\right)(j \neq i)$. Let $p_{i}$ be the polar prime of $A_{i}$ with respect to $S\left(A_{i}\right)$.

The $n+2$ primes $p_{i}$ are such that any $n+1$ of them form the $n+1$ primes $p_{j}$ of a simplex $S\left(p_{i}\right)(j \neq i)$ perspective to $S\left(A_{i}\right)$ formed by their $n+1$ corresponding points $A_{j}$. The centre and prime of perspectivity are in each case the remaining point $A_{i}$ and the remaining prime $p_{i}$. The constants of the $n+2$ perspectivities considered are equal, their common numerical value being $n+2$.

If $n=4$, as in the present case, $n+2$ is zero over $G F(3)$ and $P G(4,3)$ presents a peculiar case as distinct form that of the real projective space. In every finite space where this constant of perspectivity is zero over the corresponding finite field, we shall have similar results.

\section{A configuration of 15 points and 20 lines in $P G(3,3)$}

In the configuration of fifteen points and fifteen lines [\$1] a line contains three points of the configuration. Hence on each line of this configuration only one point is left out of the configuration. We first collect all these fifteen points. We found $[\S 1]$ that the sets of collinear points were

$$
P_{i j}, P_{l m}, A_{k}[i<j, l<m ; i, j \neq l, m \neq k]
$$

and the remaining point on this line is denoted as $P_{i j k}$ or $P_{l m k}$. The coordinates of $P_{i j k}$ or $P_{l m k}$ are such that the $k$-th coordinate is zero and the $i$-th and $j$-th coordinates are the same being negatives of the $l$-th and $m$-th coordinates. Then it is evident that the fifteen points $P_{i j k}$ lie on the solid $x_{0}+x_{1}+x_{2}+x_{3}+x_{4}$ 
$=0$ which is the polar solid of the unit point for the simplex of reference or, in other words, the polar solid of the given point $X$ for the given simplex $A_{1} A_{2} A_{3} A_{4} A_{5}[\S 1]$.

It is found that the sets of points collinear with $P_{i j k}$ are

and

$$
P_{i j k}, P_{j k l}, P_{l j i} \ldots(A)
$$

$$
P_{i j k}, P_{k i l}, P_{i l j} \ldots(B)
$$

with the understanding that $P_{i j k}, P_{j i k}, P_{l m k}, P_{m l k}$ denote the same point. Thus the points $P_{i j k}$ form a configuration in 3-space of fifteen points where each point is incident on four lines and each line contains 3 points, the number of lines in the configuration being $15 \times 4 \div 3=20$.

It is easily obtained that the number of planes through each point is six and the number of points in each plane is six. These six points in a plane form a subconfiguration of four lines, each line containing three points and each point being incident on two lines. As the number of lines through a point is four, the number of planes through a line is three. The number of planes in the configuration is $20 \times 3 \div 4=15$. In Baker's notation the configuration is

$$
15(., 4,6) 20(3, ., 3) 15(6,4, .)
$$

which implies that the configuration is self-dual.

\section{An Asymmetric Configuration}

In the configuration of fifteen points and twenty lines [ $\$ 3]$ one point on each line of the configuration is not included in it. Considering this we get only ten distinct points because at each such point two lines of the configuration meet. The pairs of lines of the set $(A)[\S 3]$ which meet at a point outside the configuration are

$$
\left.\begin{array}{lll}
P_{i j k} & P_{j k l} & P_{l j i} \\
P_{i j l} & P_{j l k} & P_{k j i}
\end{array}\right\} \quad \ldots
$$

Similarly, the pairs of lines of the set $(B)$ which meet beyond the configuration are

$$
\left.\begin{array}{lll}
P_{i j k} & P_{k i l} & P_{i l j} \\
P_{i j l} & P_{l i k} & P_{i k j}
\end{array}\right\}
$$

The coordinates of the point common to a pair of lines $(C)$ are such that $i$-th, $l$-th and $k$-th coordinates (obtained by taking last letters of the suffixes) are each unity and the other coordinates are zero. We denote such a point as $Q_{i l k}$. Obviously, any permutation of the suffixes $i, l, k$ will also denote the same point. Similarly, the point common to the pair of lines $(D)$ is $Q_{j k l}$ which is of the same type as $Q_{i l k}$. The ten points $Q_{i j k}(i, j, k=1,2,3,4,5)$ do not form a configuration. 
If we add to these ten points the five harmonic inverses of the unit point $(1,1,1,1,1)$ with respect to the vertices and opposite solids of the simplex of reference, these fifteen points will form a configuration. The harmonic inverses of the unit point with respect to the vertices $A_{i}$ and the opposite solids are denoted as $B_{i}$ $(i=1,2,3,4,5)$ where $i$-th coordinate is -1 and each of the other coordinates is 1 . The ten point $Q_{i j k}$ and the five points $B_{i}$ form a configuration of fifteen points and twenty-five lines, each line containing three points such that through a point $Q_{i j k}$ pass four lines and through a point $\mathbf{B}_{i}$ pass seven lines.

The triads of collinear points are

and

$$
\left.\begin{array}{l}
Q_{i j k}, Q_{l m n}, B_{n} \\
Q_{i j k}, B_{l}, B_{m}
\end{array}\right\} \text { collinear with } Q_{i j k}
$$

$$
\left.\begin{array}{l}
B_{l}, B_{m}, Q_{i j k} \\
B_{l}, Q_{l i j}, Q_{l k m}
\end{array}\right\} \text { collinear with } B_{l}
$$

where $i \neq j \neq k \neq l \neq m ; n=i, j, k$,

$$
i, j, k, l, m=1,2,3,4,5 \text {. }
$$

A configuration of this type will be called, due to want of usual symmetry, an asymmetrie configuration. These fifteen points lie on the solid

$$
x_{0}+x_{1}+x_{2}+x_{3}+x_{4}=0
$$

which is the polar solid of the unit point for the simplex of reference.

\section{Chain of Configurations}

It is seen that starting with a given simplex in $P G(4,3)$ and a point not lying in any face of it we get successively three configurations forming a chain. First, a Segre configuration of fifteen points and fifteen lines in 4-spaces, secondly, a self-dual configuration of fifteen points and twenty lines in 3-space and lastly, an asymmetric configuration of fifteen points and twenty-five lines in 4-space.

It is interesting to note that considering harmonic inverses of the same point with respect to two suitably chosen different 4 -simplexes in $P G(4,3)$ we get two chains of configurations which differ only in the first configuration, the second and last configurations being the same.

For example, we consider the harmonic inverses of the unit point with respect to the edges and opposite planes of the simplex $\Sigma$ whose vertices are

$$
P_{1}(0,1,1,1,1), P_{2}(1,0,1,1,1), P_{3}(1,1,0,1,1), P_{4}(1,1,1,0,1) \text { and } P_{5}(1,1,1,1,0) \text {. }
$$

If $R_{i j}$ be the harmonic inverse of the unit point with respect to the edge $P_{i} P_{j}$ of $\Sigma$ and the opposite plane, then each of $i$-th and $j$-th coordinates of $R_{i j}$ is unity and the other coordinates are zero. 
Then, as proved in $\S 1$ the five vertices $P_{i}(i=1,2,3,4,5)$ of $\Sigma$ and the ten harmonic inverses $R_{i j}(i<j) ; i, j=1,2 \ldots 5$ form a Segre configuration of fifteen points and fifteen lines. This configuration gives rise to a self-dual configuration of fifteen points and twenty lines in 3-space which again leads to an asymmetric configuration of fifteen points and twenty-five lines in 4-space. It is seen that the points of the above self-dual and asymmetric configurations are the same as those obtained in $\S \S 3$ and 4 emanating from a different Segre configuration.

It may be noted here that the harmonic inverses of the given point i.e. the unit point with respect to the vertices and the opposite solids of the simplex of reference and the simplex $\Sigma$ are the same. In fact, this forms the basis on which the two different simplexes are to be chosen to obtain a pair of chains having the required property.

My sincere thanks are due to Dr. Sahib Ram Mandan for his help and guidance in prepering this paper. I am grateful to the referee for his constructive suggestions in presenting the paper in this form.

\section{References}

[1] H. F. Baker, Principles of Geometry, Vol. 4 (Cambridge, 1940).

[2] W. L. Edge, 'Geometry in three dimensions ever GF (3)' Proc. Roy, Soc., London, Vol.222A (1954)

[3] S. R. Mandan, 'Court's Conjecture on $n+2$ points in [n]', Casepis Pre Pěstováni matematiky, roè. 90 (1965), Praha.

[4] S. R. Mandan, 'Polarity for a Simplex,' Чехосдовацкнй математически журнал, т. I6 (I966), 9r Прага.

I. C. V. Polytechnic, Jhargram

Sevayatan

Midnapore

West Bengal, India 